\title{
Management Strategy of Isolated Spontaneous Dissection of the Superior Mesenteric Artery
}

\author{
Hirono Satokawa, MD, PhD, ${ }^{1}$ Shinya Takase, MD, PhD, ${ }^{1}$ Yuki Seto, MD, PhD, ${ }^{1}$ \\ Hitoshi Yokoyama, MD, PhD, ${ }^{1}$ Mitsukazu Gotoh, MD, PhD, ${ }^{2}$ \\ Michihiko Kogure, MD, PhD, ${ }^{2}$ Hirofumi Midorikawa, MD, PhD, ${ }^{3}$ \\ Tomiyoshi Saito, MD, PhD, ${ }^{4}$ and Kazuhira Maehara, MD, PhD ${ }^{4}$
}

\begin{abstract}
Objective: Isolated spontaneous dissection of the superior mesenteric artery (SMA) is very rare among of the visceral artery dissection and its treatment is not established. In this paper we present our experiences and consider the treatment of isolated SMA dissection.

Methods: A retrospective review of our cases from 2005 was performed. Clinical symptoms, radiologic findings and results were evaluated. There were 14 cases of visceral artery dissection, in which all cases were with SMA dissection. There were 12 males and 2 females with a mean age of 57 years (range 41-78 years).

Results: We categorized SMA dissection into the six types according to the Sakamoto's and Zerbib's classification. One patient with type VI underwent emergent endovascular surgery with stent. One patient with type VI received thrombectomy and intimectomy with open surgery. One patient with type II underwent aneurysmectomy due to enlarged dissected SMA 3 months later from onset. The other eleven patients were managed conservatively. At follow-up, the diameter of SMA did not enlarged and the length of the dissection significantly decreased to $20.7 \pm 15.7 \mathrm{~mm}$ from $38.0 \pm 15.1 \mathrm{~mm}$ at onset $(p<0.01)$. After treatment, imaging indicated the following changes in classification: type I, one patient; type II, 4 patients; type IV, 4 patients; complete remodeling, one patient, all without any event during the follow-up period of 5-82 months.

Conclusion: Most patients with isolated visceral artery dissection occurred in superior mesenteric artery and can be treated conservatively; however, endovascular or surgical procedures including laparotomy are indicated when there is suspicion of severe mesenteric ischemia. Because the dissection configuration will change, long term follow-up is necessary. (*English translation of Jpn J Vasc Surg 2013; 22: 695-701)
\end{abstract}

Keywords: visceral artery dissection, spontaneous isolated dissection of superior mesenteric artery, endovascular treatment, abdominal open surgery

${ }^{1}$ Department of Cardiovascular Surgery, Fukushima Medical University, School of Medicine, Fukushima, Fukushima, Japan

${ }^{2}$ Department of 1st Surgery, Fukushima Medical University, School of Medicine, Fukushima, Fukushima, Japan

${ }^{3}$ Department of Cardiovascular Surgery, Minami Toboku General Hospital, Kooriyama, Fukushima, Japan

${ }^{4}$ Department of Circulatory Internal Medicine, Shirakawa

Kosei General Hospital, Shirakawa, Fukushima, Japan

Received: June 11, 2014; Accepted: June 11, 2014

Corresponding author: Hirono Satokawa, MD. Department of Cardiovascular Surgery, Fukushima Medical University, School of Medicine, 1 Hikarigaoka, Fukushima, Fukushima 960-1295, Japan

Tel: +81-24-547-1111, Fax: +81-24-548-3926

E-mail: satokawa@fmu.ac.jp

*This article is English translation of Jpn J Vasc Surg 2013; 22: 695-701.

\section{Introduction}

Isolated visceral artery dissection (VAD) not accompanied by aortic dissection develops with sudden abdominal pain in many cases, mostly in the superior mesenteric artery (SMA). It is a rare disease, but has recently become frequently discovered because of the development of computed tomography (CT); however, its pathology remains unclear. ${ }^{1}$ Although there are considered to be many causative factors of the disease, treatment methods have not been established.

In this study, we report VAD patients whom our laboratory encountered, with SMA dissection patients accounting for the majority, along with a literature review. 


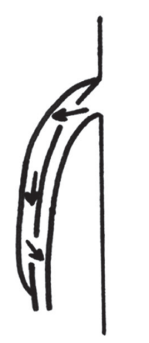

Type I

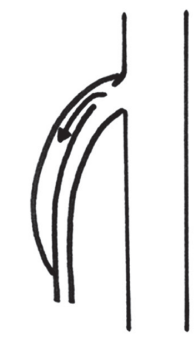

Type II

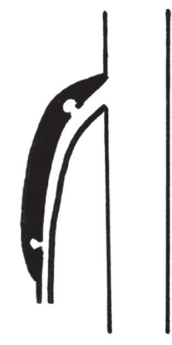

Type III

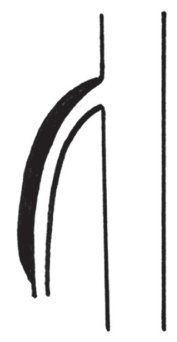

Type IV

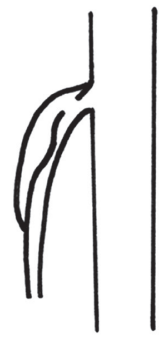

Type V

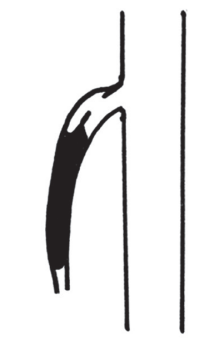

Type VI

Fig. 1 Modified Sakamoto's classification of isolated superior mesenteric artery dissection.

\section{Subjects and Methods}

The treatment methods, outcomes, complications, and long-term follow-up results were investigated in VAD patients whom our laboratory encountered.

Sakamoto, et al. ${ }^{2)}$ classified the morphology of isolated SMA dissection into 4 types: type I, patent type with an entry and re-entry; type II, "cul-de-sac" type without re-entry; type III, with a thrombosed false lumen with ulcer-like projection (ULP); and type IV, with a completely thrombosed false lumen without ULP. Zerbib, et al. ${ }^{3)}$ added type V accompanied by dissection and stenosis of the SMA and type VI with partial or complete occlusion (Fig. 1). The dissection morphologies of our patients were typed and compared following this classification.

The values are presented as the mean \pm standard deviation and were compared using the paired t-test. A p-value below 0.05 was regarded as significant.

\section{Results}

At our laboratory, $14 \mathrm{VAD}$ patients have been encountered since 2005 . Their ages were $41-78$ years old (mean: 56.7 years old), and there were 12 males and two females. Splenic artery was concomitantly present in one patient, but all patients had SMA dissection. One patient was complicated by abdominal aortic aneurysm with a maximum diameter of $35 \mathrm{~mm}$. Regarding background factors, nine patients had hypertension and 12 patients were cigarette smokers. VAD was incidentally discovered on diagnostic CT for other diseases in five patients. It developed with sudden abdominal pain in the other nine patients and bloody stool was noted in three of them (Table 1).

The dissection morphology was typed based on the classification established by Sakamoto, et al. The type was I in two, II in one, III in two, IV in six, V in one, and VI in two patients. Abdominal pain was noted in 1, 1, 1, 4, and 2 cases of types I-VI, respectively. The SMA diameter measured at the onset time on CT was 8-12 (mean: 9.2) mm, suggesting that the risk of acute-phase rupture was low. In treatment, cases of type VI were treated urgently, considering the possibility of intestinal ischemia. The SMA was dilated and formed an aneurysm in the type II patient and surgery was performed 3 months later. The other cases are presented below:

Patient 5 was a 41-year-old male. Severe abdominal pain persisted for about 8 hours, and complete occlusion of the SMA extending over $15 \mathrm{~cm}$ and marked intestinal edema were noted on CT, for which urgent reperfusion of the SMA was considered necessary. We asked the Department of Cardiovascular Medicine for direct insertion of SMART stents, $10 \mathrm{~mm} \times 60 \mathrm{~mm}$ and $6 \mathrm{~mm} \times 100 \mathrm{~mm}$ (Cordis, Tokyo, Japan), and recanalization was achieved. Postoperative paralytic ileus developed, but it was remitted by conservative treatment and the patient was discharged. After surgery, medication with oral cilostazol (Pletaal, Otsuka Pharmaceutical, Tokushima, Japan) was continued (Fig. 2).

Patient 9 was a 53-year-old male. The disease suddenly developed with abdominal pain. On CT, dissection and complete occlusion of the SMA extending over about $20 \mathrm{~cm}$ were noted. Since abdominal pain persisted and abdominal muscular defense was observed, intestinal ischemia was suspected, and emergency laparotomy was performed at the Department of Gastrointestinal Surgery 4 hours after the onset, for which our department was asked for help. Since the pulse of the SMA was absent, an approximately 7-8 mm longitudinal incision was made, thrombointimectomy was applied, and reperfusion of the SMA was achieved. The intestine was not treated 


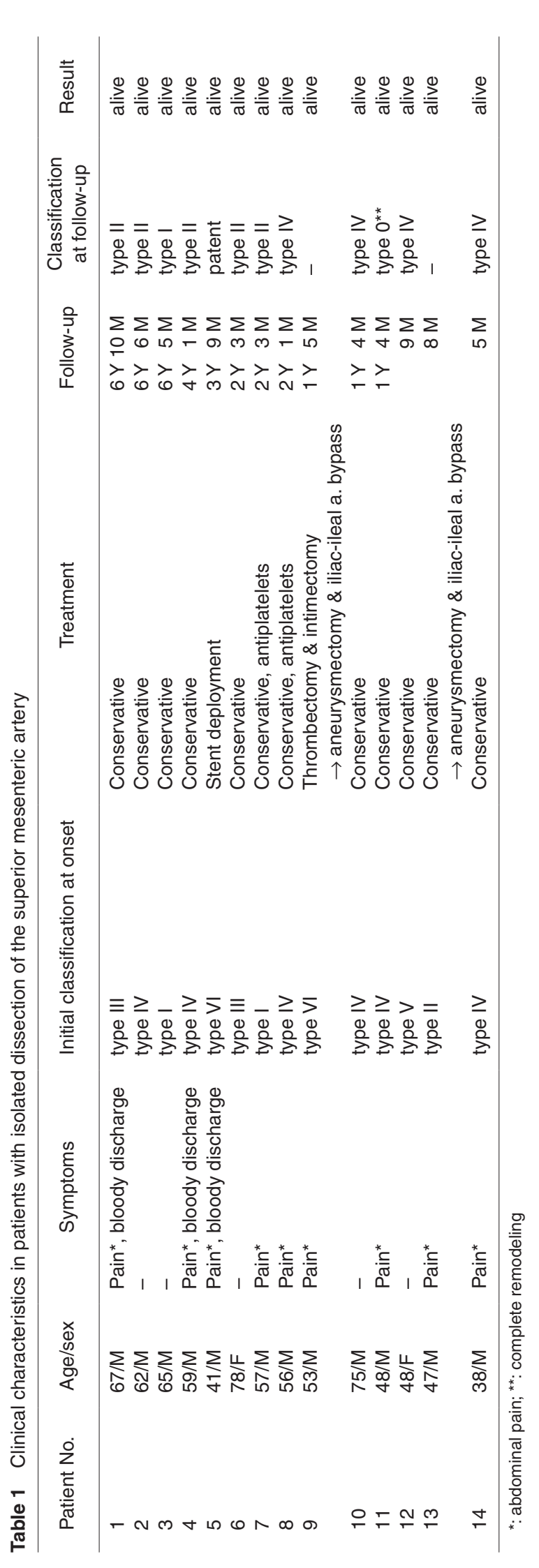

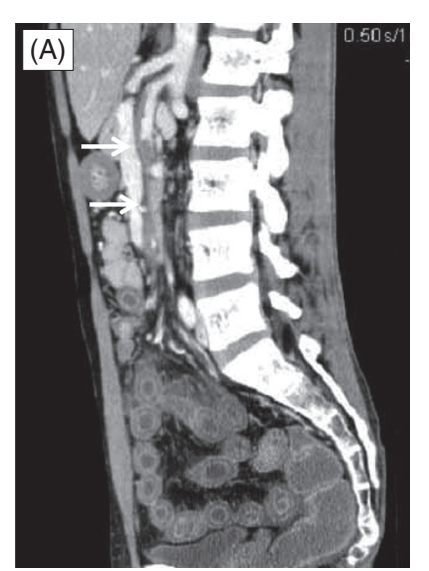

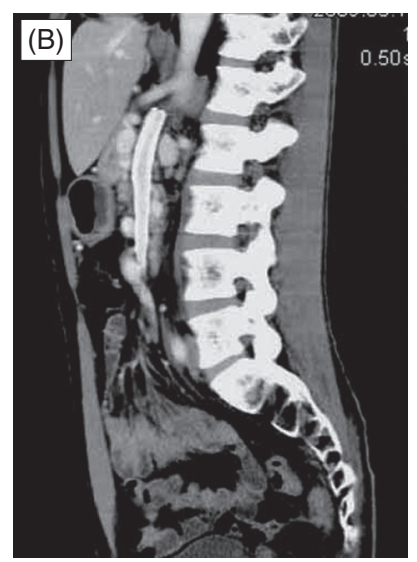

Fig. 2 Computed tomography (CT) of case 5. (A) Preoperative CT (sagittal view) showing the occluded superior mesenteric artery (white arrow) and severe edema of intestine. (B) CT at 3 years from stent deployment.

because no intestinal necrosis or unfavorable color was noted. However, on follow-up CT performed after 5 months, the SMA diameter had increased to $40 \mathrm{~mm}$ in the surgically treated region, forming an aneurysm, for which surgery was performed in consideration of the risk of rupture. The SMA aneurysm was resected through re-application of an abdominal midline incision. The right colic and ileocolic arteries were ligated because they were thin and backflow was noted, and bypass from the iliac artery to the peripheral SMA was prepared with an autologous vein. ${ }^{4}$

Patient 13 was a 47-year-old male admitted for disease onset with rapid abdominal pain. SMA dissection was diagnosed on CT, and conservative course observation was selected. Symptoms improved thereafter and the patient was discharged. The SMA morphology at the onset time was type II with a diameter of $9 \mathrm{~mm}$, but the diameter increased to a maximum of $39 \mathrm{~mm} 3$ months later. Marked expansion of the false lumen and compression of the true lumen were noted in the aneurysm region. It was considered difficult to apply endovascular treatment based on the morphology and to conserve peripheral blood flow. Laparotomic aneurysmectomy was performed, and bypass to the peripheral SMA was prepared with an autologous vein (Fig. 3).

The other 11 patients were conservatively treated. Course observation with fasting was selected for patients with abdominal pain, and aspirin (Bayaspirin, Bayer Pharmaceutical, Osaka, Japan) was administered for 3 months to two patients without bloody stool. The dissection was incidentally discovered in 

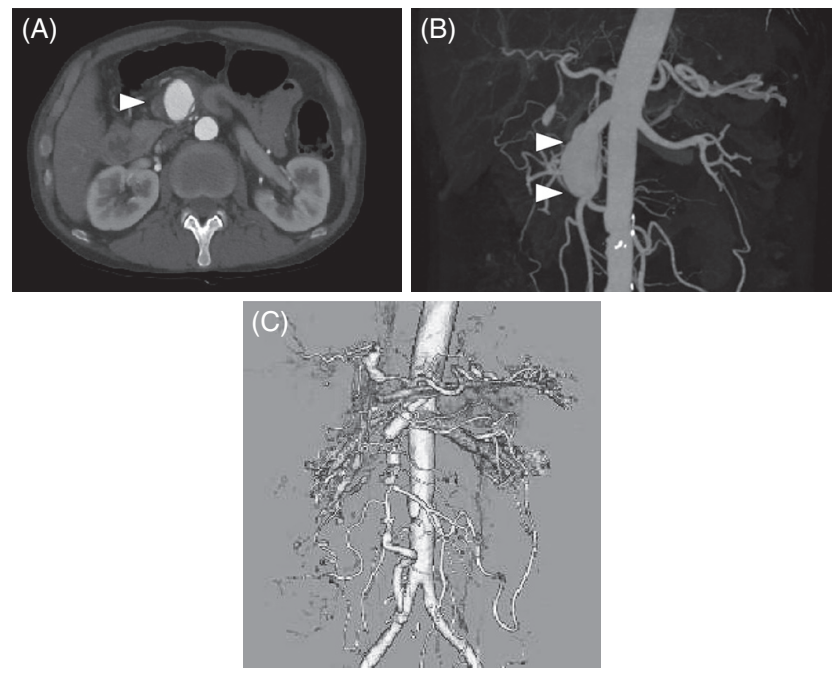

Fig. 3 Computed tomography (CT) of case 13. (A, B) The white angle showed an aneurysm of the superior mesenteric artery at 3 months later from onset, (C) CT showing iliacileal artery bypass after aneurysmectomy.

five patients, and only course observation was performed in all of these. Digestive symptoms improved in all conservatively treated patients, and they were discharged.

The duration of follow-up ranged from 5 months to 6 years and 10 months (mean: 2 years and 10 months), and the follow-up rate was $100 \%$. The stented patient was followed for 3 years and 9 months after deployment, and patency was maintained. One year and 5 months and 8 months had passed in the 2 patients who received direct surgery. The morphology of the dissection on CT was investigated over long-term follow-up in the 11 conservatively treated patients. The maximum SMA diameter measured on CT was $9.7 \pm 1.5 \mathrm{~mm}$ in the acute phase and $8.9 \pm 1.6 \mathrm{~mm}$ after long-term follow-up, showing no significant dilation. The length of dissection was $38.0 \pm 15.1 \mathrm{~mm}$ at the onset time and $20.7 \pm 15.7 \mathrm{~mm}$ after long-term follow-up, showing a significant reduction $(\mathrm{p}<0.001)$. Morphologically, type II and IV cases increased with the reductions of the false lumen and thrombus over long-term follow-up, 1, 5, and 4 cases improved to types I, II, and IV, respectively, and complete resolution was noted in one. In patients followed for more than 2 years, the size of thrombosed false lumen decreased, and many cases became a type-II small aneurysm. No digestive complications developed in any patient throughout the follow-up period, and all patients are alive at the time of writing.

\section{Discussion}

Visceral artery dissection (VAD) is very rare $^{1)}$ and mostly develops in the SMA. Mousa, et al..$^{5)}$ identified 71 cases of SMA dissection and 12 cases of celiac artery dissection reported in the literature, and found that the patients were mostly male and young, with a mean age of 55 years old. Similarly, VAD involved SMA dissection in all of our patients, and one patient was accompanied by celiac artery dissection. Thus, SMA dissection is mainly discussed below.

Regarding the cause of SMA dissection, associations with hypertension and cigarette smoking have been pointed out, ${ }^{6}$ and these were also noted in $58 \%$ and $83 \%$ of our patients, respectively. For isolated SMA dissection, histologically, arteriosclerosis, cystic medial necrosis, abnormalities of elastic fiber (Marfan syndrome and Ehlers-Danlos syndrome), trauma, and pregnancy are considered as risk factors. ${ }^{5)}$ In one of our surgically treated patients, histological mucous degeneration and destruction of the media intima were noted, suggesting that cystic medial necrosis was the cause; however, generally, the cause is unclear in many cases. In aortic dissection, the DeBakey classification type II with an entry into the ascending aorta is the most common type, accounting for $38 \%$, and hypertension, aortic dilation, and fragility of the wall are considered as the causes. ${ }^{7)}$ In contrast, for SMA dissection, a mechanical influence is considered to be the cause based on the structural characteristics of the SMA. The entry of SMA dissection is produced at $3-4 \mathrm{~cm}$ from the root in most cases. ${ }^{8)}$ Since this region is adjacent to the lower surface of the pancreas, large shear stress is loaded on the vascular wall, and this is considered to influence the development of dissection. ${ }^{5}$ Dissection occurred from this region in most of our patients.

SMA dissection is accompanied by abdominal pain in many cases, and vascular murmur is heard, ${ }^{8)}$ but many asymptomatic cases are incidentally discovered. Since there are no specific symptoms, it is difficult to make a conclusion based on symptoms and special examination, and diagnostic imaging is necessary to make a definite diagnosis, for which ultrasonography, contrast CT, CT-angiography, MRI, and angiography are used. The resolution of CT has recently markedly improved, and the condition of not only dissection but also the surrounding tissue and intestine can be examined, ${ }^{6}$ being very useful for the 
diagnosis and follow-up. In particular, CT-angiography is very useful to diagnose this disease because the condition of dissection can be readily diagnosed compared with the case using angiography, ${ }^{9}$ facilitating selection of a treatment method corresponding to the pathology. The indication of angiography may be limited in the future, such as for endovascular treatment.

The SMA dissection morphology was classified as described above by Sakamoto, et al. ${ }^{2}$ and Zerbib, et al. ${ }^{3)}$ Yun, et al. ${ }^{10)}$ classified the morphology into type I (the same as that classified by Sakamoto, et al.), type II (cases with no re-entry, the false lumen is patent [IIa] and occluded [IIb]), and type III (occluded). We adopted the more detailed classification established by Sakamoto, et al. and Zerbib, et al. It is considered desirable to perform active treatment, such as surgical and endovascular treatments, when the diameter is $2 \mathrm{~cm}$ or greater or stenosis is $70 \%$ or more in cases of types II, III, V and VI of this classification. ${ }^{3)}$ Among our patients, intestinal ischemia was suspected in a type VI patient with long occlusion, who was urgently treated. When the summation reported by Park, et al. ${ }^{11)}$ was applied to the classification reported by Sakamoto, et al., type I cases with an entry and re-entry and a patent false lumen were the most common, accounting for $39 \%$, type II without re-entry accounted for $21 \%$, types III and IV accounted for $35 \%$, and type VI accounted for $4 \%$. Generally, the frequency of the type with an occluded false lumen is low in aortic dissection. Compared with this, the false lumen may be closed in many cases in SMA dissection because re-entry was formed only in a few cases.

Treatment of SMA dissection includes conservative, endovascular, and surgical treatments. Conservative treatment includes intestinal rest by fasting and internal medical treatment. In internal medical treatment, in addition to blood pressure control, anticoagulant, ${ }^{8)}$ antiplatelet, and thrombolytic therapies $\left.{ }^{6}\right)$ are performed. Anticoagulant therapy does not inhibit the progression of dissection, ${ }^{12)}$ but it was reported also to be effective for severe stenosis $(90 \%)$ of the root of the SMA. ${ }^{13)}$ Conservative treatment was reported to be effective for $55 \%-63 \%$ of SMA dissection cases, ${ }^{14)}$ but invasive treatment was necessary for $8.6 \%-34.8 \%$ because of intestinal ischemia and aneurysm enlargement. ${ }^{11,15)}$ The absolute indications of emergency surgical treatment for SMA dissection are intestinal ischemia and rupture. ${ }^{6}$ Intestinal ischemia is likely to occur in the acute phase after the onset due to occlusion and stenosis of the SMA false lumen induced by the progression of dissection. Abdominal pain accompanying SMA dissection is not necessarily consistent with ischemia, and it is difficult to diagnose intestinal ischemia based on symptoms alone. The features of intestinal ischemia on CT are poor enhancement of the intestinal wall, edema of the mesentery, and gas in the mesenteric blood vessels in advanced cases. However, even though SMA dissection can be diagnosed, it is difficult to identify whether or not ischemia of the organ occurs. Min, et al. ${ }^{15)}$ stated that endovascular treatment should be attempted when the true lumen is $80 \%$ or more stenosed or dilated to $2.0 \mathrm{~cm}$ or more, suggesting intestinal ischemia. Subhas, et al. ${ }^{6}$ proposed a treatment algorithm for SMA dissection including diagnostic laparoscopy to ensure the presence of intestinal ischemia. Trial laparoscopy for an emergency is not common in actual medical practice, and Katsura, et al. recommended trial laparotomy. ${ }^{16)}$ If endovascular treatment improves intestinal ischemia, it should be selected because it has low invasiveness compared with laparotomy, but when severe ischemia including intestinal necrosis is suspected, or a large volume of hemorrhage is present, trial laparotomy enabling observation of the intestine and surgical treatment should be selected in consideration of surgery (Fig. 4).

Many surgical treatment procedures for SMA dissection have been reported, including endarterectomy, fixation of the media intima, patching, aneurysmorrhaphy, aorta-SMA bypass, gastroepiploic artery-SMA bypass, and SMA-aorta end-to-side anastomosis. ${ }^{11,15-17)}$ In one of our patients treated with surgery for suspected intestinal ischemia, thrombo-intimectomy was applied and prevented intestinal ischemia, but it would have been better first to apply endovascular treatment. To realize this, it may be necessary to cooperate closely with physicians performing endovascular treatment and with ones doing gastrointestinal surgery for emergency. For the other patient in whom false aneurysm enlarged in the subacute phase, direct surgery was selected because it was concluded that endovascular treatment would be morphologically difficult to apply.

More than 10 cases of endovascular treatment for SMA dissection ${ }^{2,6,15)}$ have been reported since Leung, et al. treated one case with stenting in $2000 .^{14)}$ 


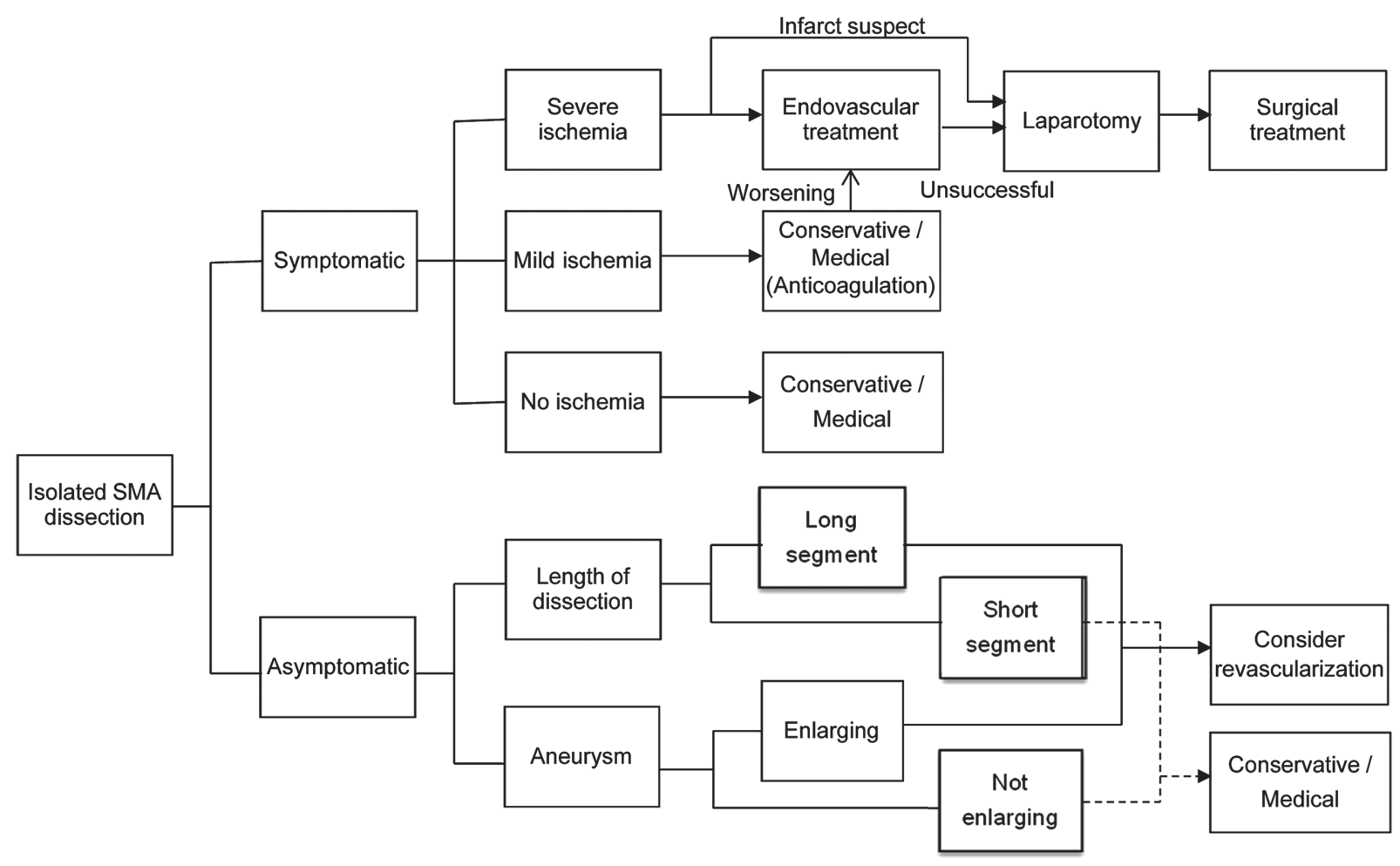

Fig. 4 Treatment algorithm for isolated superior mesenteric artery dissection.

Endovascular treatment includes thrombolysis, thrombus suction, balloon dilation, stent graft placement, and stenting, the latter of which has recently been increasingly applied. Some reports recommend stent graft, but a risk of occlusion of branches has been pointed out. Endovascular treatment has low invasiveness and rapidly improves ischemia, shortening the hospital stay. However, it is disadvantageous in that the intestine cannot be directly observed and there is a risk for arterial rupture. ${ }^{11)}$ The duration of follow-up after endovascular treatment was short (3-38 months), 6) but it may be initially selected if the long-term outcome is favorable.

Regarding rupture of SMA dissection, rupture in the acute phase has rarely been reported. In another report, no false aneurysm developed over long-term follow-up. ${ }^{11)}$ However, an aneurysm may enlarge after the acute phase, such as was observed in Patient 13 and reported cases of rupture after long-term followup, ${ }^{18)}$ to which attention should be paid. Since the dissection morphology changes with time, periodic examination by CT is recommended. ${ }^{2)}$ Follow-up of the dissection morphology and aneurysm enlargement may be important. Park, et al. ${ }^{11)}$ reported that reductions of the false lumen diameter and dissected region were noted in $41.3 \%$ and $23.9 \%$ of conservatively treated patients after 23-month long-term follow-up, respectively, and dissection did not progress in any patient. In our patients who transited to the chronic phase, the false lumen size decreased, and cases of types III and IV in the acute phase changed to type II small aneurysms in the chronic phase. False aneurysm enlarged after 3 months in one patient, but no significant enlargement of the false lumen or progression of stenosis was noted over the long-term follow-up in any other patient. However, the follow-up is still insufficient because the maximum duration of long-term follow-up was 4 years in reported cases. ${ }^{19}$ ) After the onset, periodic observation is necessary, and follow-up should be continued until dissection is completely resolved.

\section{Conclusion}

Isolated visceral artery dissection mostly develops in the SMA and is considered very rare, but its incidence has been increasing. Many cases of isolated SMA dissection are improved by conservative treatment, but, when intestinal ischemia is suspected, endovascular treatment and surgery including trial laparotomy 
should be selected. Periodic observation not only in the acute but also chronic phase and follow-up of the dissection morphology are important.

\section{Disclosure Statement}

The authors have no conflict of interest to declare.

\section{References}

1) Takayama T, Miyata T, Shirakawa M, et al. Isolated spontaneous dissection of the splanchnic arteries. J Vasc Surg 2008; 48: 329-33.

2) Sakamoto I, Ogawa Y, Sueyoshi E, et al. Imaging appearances and management of isolated spontaneous dissection of the superior mesenteric artery. Eur J Radiol 2007; 64: 103-10.

3) Zerbib P, Perot C, Lambert M, et al. Management of isolated spontaneous dissection of superior mesenteric artery. Langenbecks Arch Surg 2010; 395: 437-43.

4) Satokawa H, Seto Y, Yamamoto A, et al. A case report of aneuysmectomy after thrombo-intimectomy for spontaneous isolated superior mesenteric artery dissection. Ann Vasc Dis 2012; 5: 204-7.

5) Mousa AY, Coyle BW, Affuso J, et al. Nonoperative management of isolated celiac and superior mesenteric artery dissection: case report and review of the literature. Vascular 2009; 17: 359-64.

6) Subhas G, Gupta A, Nawalany M, et al. Spontaneous isolated superior mesenteric artery dissection: a case report and literature review with management algorithm. Ann Vasc Surg 2009; 23: 788-98.

7) 2010 JCS Joint Working Groups for Guidelines for Diagnosis and Treatment of Cardiovascular Diseases. Guidelines for Diagnosis and Treatment of Aortic Aneurysm and Aortic Dissection (JCS 2011).

8) Sparks SR, Vasquez JC, Bergan JJ, et al. Failure of nonoperative management of isolated superior mesenteric artery dissection. Ann Vasc Surg 2000; 14: 105-9.
9) Furukawa H, Moriyama N. Spontaneous dissection of the superior mesenteric artery diagnosed on multidetecter helical CT. J Comput Assist Tomogr 2002; 26: 143-4.

10) Yun WS, Kim YW, Park KB, et al. Clinical and angiographic follow-up of spontaneous isolated superior mesenteric artery dissection. Eur J Vasc Endovasc Surg 2009; 37: 572-7.

11) Park YJ, Park KB, Kim DI, et al. Natural history of spontaneous isolated superior mesenteric artery dissection derived from follow-up after conservative treatment. J Vasc Surg 2011; 54: 1727-33.

12) Krupski WC, Effeney DJ, Ehrenfeld WK. Spontaneous dissection of the superior mesenteric artery. J Vasc Surg 1985; 2: 731-4.

13) Nozu T, Komiyama H, Okumura T. Image of the month. Isolated dissection of the superior mesenteric artery. Gastroenterology 2004; 127: 1029, 1282.

14) Leung DA, Schneider E, Kubik-Huch R, et al. Acute mesenteric ischemia caused by spontaneous isolated dissection of the superior mesenteric artery: treatment by percutaneous stent placement. Eur Radiol 2000; 10: 1916-9.

15) Min SI, Yoon KC, Min SK, et al. Current strategy for the treatment of symptomatic spontaneous isolated dissection of superior mesenteric artery. J Vasc Surg 2011; 54: 461-6.

16) Katsura M, Mototake $H$, Takara H, et al. Management of spontaneous isolated dissection of the superior mesenteric artery: case report and literature review. World J Emerg Surg 2011; 6: 16.

17) Morris JT, Guerriero J, Sage JG, et al. Three isolated superior mesenteric artery dissections: update of previous case reports, diagnostics, and treatment options. J Vasc Surg 2008; 47: 649-53.

18) Nomura Y, Yamaguchi M, Kitagawa A, et al. Hybrid management of ruptured isolated superior mesenteric artery dissecting aneurysm. J Vasc Surg 2011; 54: 1808-11.

19) Nakamura K, Nozue M, Sakakibara Y, et al. Natural history of a spontaneous dissecting aneurysm of the proximal superior mesenteric artery: report of a case. Surg Today 1997; 27: 272-4. 